\title{
Abnormal Liver Function Tests of Patients with Coronavirus Disease 2019 in Mainland China: A Systematic Review and Meta- Analysis
}

\author{
Shengliang Xin, Jinghang Xu, Yanyan Yu
}

Department of Infectious

Disease, Peking University

First Hospital, Beijing,

China

\begin{abstract}
Aims: Comparing the risk of abnormal liver function tests between severe and non-severe patients with coronavirus disease 2019 (COVID-19) by meta-analysis.

Methods: A literature search was conducted using the databases PubMed, Embase, and Cochrane Library. Odds ratios (ORs) and 95\% confidence intervals (CIs) were pooled using fixed- or random-effects models. Publication bias was detected by the Harbord test.

Results: We included 8 articles comprising 7,467 COVID-19 patients. When compared between severe and non-severe COVID-19 patients, the pooled ORs of elevated alanine aminotransferase, aspartate aminotransferase, total bilirubin, and lactate dehydrogenase levels were 2.35 (95\% CI 1.38-3.98), 3.21 (95\% CI 2.59-3.98), 1.87 (95\% CI 1.32-2.65), and 4.83 (95\% CI 2.90-8.05), respectively.

Conclusions: The severity of COVID-19 is associated with liver damage, and can be a risk factor for abnormal liver function tests.
\end{abstract}

Key words: COVID-19 - liver - laboratory tests - meta-analysis.

\begin{abstract}
Abbreviations: ACE2: angiotensin-converting enzyme 2; ALT: alanine aminotransferase, AST: aspartate aminotransferase; COVID-19: coronavirus disease 2019; IL-6: interleukin-6; LDH: lactate dehydrogenase; NF-kB: nuclear factor - kB; SARS-CoV-2: severe acute respiratory syndrome coronavirus-2; STAT: signal transducer and activator of transcription; TBil: total bilirubin.
\end{abstract}

\section{INTRODUCTION}

The pandemic of coronavirus disease 2019 (COVID-19) was started with a few unexplained pneumonia patients in Wuhan, China in early December 2019. In the next three months, COVID-19 swept all over the China. The International Committee on the Taxonomy of Viruses named the pathogen as severe acute respiratory syndrome coronavirus-2 (SARS-CoV-2). A total of 84,565 confirmed cases, including 4,645 deaths had been reported as of May 30, 2020, by the National Health Commission of the People's Republic of China. Now, the epidemic is under effective control in China. However, in parallel, COVID-19 had spread to other parts of the world. Currently, there are 6.05 million confirmed cases globally. The real-time fatality rate is $24.52 \%, 18.99 \%$ and $16.21 \%$ in Yemen Republic, France and Belgium, respectively. The common clinical presentations of COVID-19 are fever (91.3\%), cough (67.7\%), fatigue (51.0\%), and dyspnea (30.4\%) [1]. In addition, liver damage was found among COVID-19 patients; however, the incidence rates were inconsistent. Chen et al. [2] reported in Lancet that of 99 COVID-19 patients in Wuhan Jinyintan Hospital, 43 patients (43.4\%) had different degrees of liver damage, including one with severe liver injury. Qian et al. [3] summarized the laboratory tests of 324 COVID-19 patients in Shanghai, China and found that the percentage of abnormal liver function tests was low and the degree of liver damage was mild. Zhu et al. [4] conducted a meta-analysis on 3,062 COVID-19 patients, which indicated that the incidence of liver dysfunction was $29 \%$. However, the relationship between liver damage and COVID-19 severity is unclear. Therefore, we performed this meta-analysis on published clinical studies in mainland China, and compared the risk of abnormal liver function between severe and non-severe COVID-19 patients. 


\section{METHODS}

All relevant articles on the laboratory findings of COVID-19 patients available on multiple electronic databases including PubMed, Embase, and Cochrane library up to April 22,2020 were searched. The retrieval strategy is shown in the Supplementary File. Language was not limited. Additional literature was not included in the study. We collected 506 records according to the eligibility criteria. The preliminary screening process (title and abstract screening) was performed by two authors (S.L.X. and J.H.X.) independently and blindly. The second screening process (full-text review) was performed by all authors. Finally, 8 articles containing 7,467 cases were included according to the eligibility criteria.

We included articles that met the following conditions: (1) COVID-19 confirmed cases; and (2) laboratory tests included alanine aminotransferase (ALT), aspartate aminotransferase (AST), bilirubin, and lactate dehydrogenase (LDH). We excluded articles with the following conditions: (1) duplicated records; (2) reviews, editorials, case reports, letters, and familybased studies; (3) outside mainland China studies; (4) those which did not divide COVID-19 patients into severe and nonsevere cohorts; and (5) which did not provide the numbers and proportions of COVID-19 patients with abnormal liver function tests in severe and non-severe cohorts.

We constructed $2 \times 2$ contingency table and extracted the number of severe COVID-19 patients with normal and abnormal liver function parameters and non-severe COVID-19 patients with normal and abnormal liver function parameters in each study.

Stata SE version 15 was used to perform the meta-analysis. The first step was to calculate pooled odds ratio (OR). $\mathrm{I}^{2}$ statistic was used to evaluate the heterogeneity among studies. $\mathrm{I}^{2}>50 \%$ indicated a considerable heterogeneity, thus, random-effects model was used. The second step was to assess publication bias. The Harbord test was applied to examine the potential publication ( $\mathrm{p}$ value $<0.05$ suggests a significant publication bias).

\section{RESULTS}

The literature retrieval yielded 506 records. Using Endnote X9, duplicate records $(n=167)$ and non-target article types (e.g., review, comment, case report, letter, family-based study) ( $\mathrm{n}=189$ ) were excluded. During the preliminary screening process (title and abstract screening), we eliminated irrelevant articles $(n=4)$ and experimental studies $(n=20)$. During the second screening process (full-text review), we included eight articles that met the eligibility criteria. A flow diagram of the literature selection is presented in Fig 1.

The meta-analysis was conducted on 25 tests included in the eight articles. Given that a sufficient number of tests was required for meta-analysis, we included the parameters ALT, AST, total bilirubin (TBil) and LDH in this study. We extracted the basic characteristics (e.g., first author, date, proportion of males, median age, sample size, laboratory findings) of each article (Table I) [5-12]. Moreover, previous liver comorbidities and drug administration of the included COVID-19 patients were listed in Table II. In our study, all

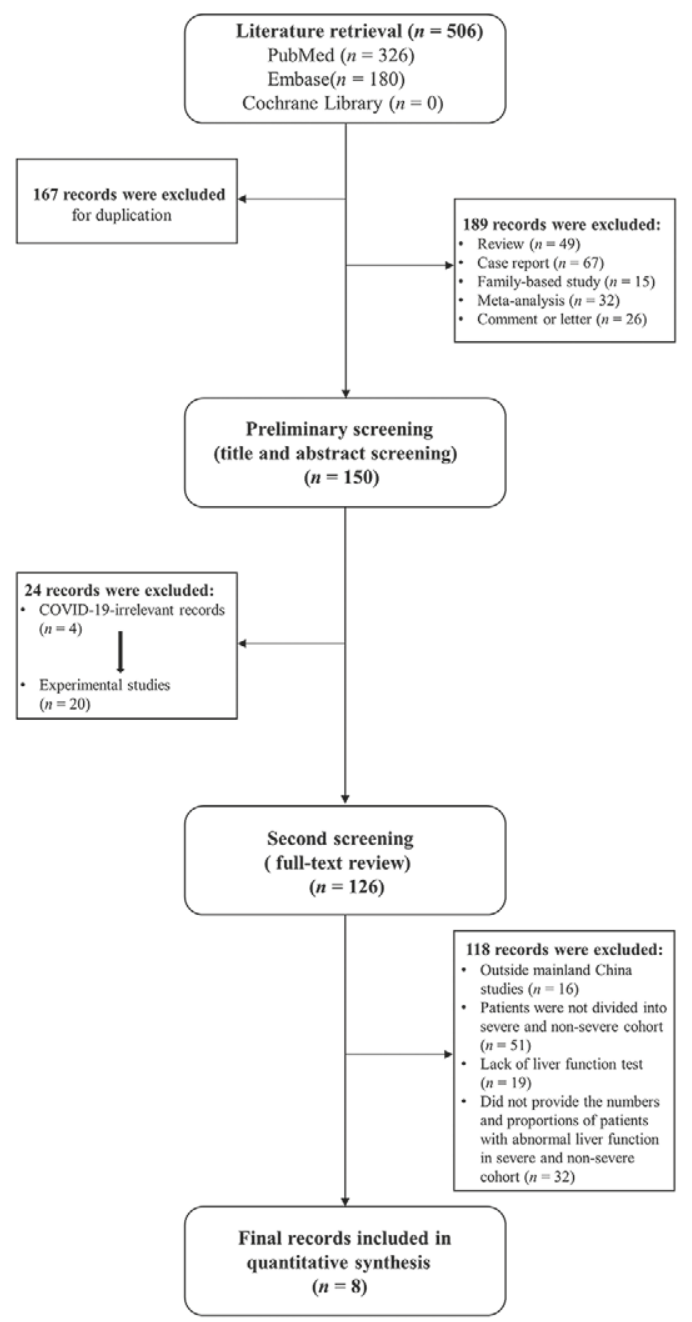

Fig. 1. Article selection process.

COVID-19 patients had tested positive for SARS-CoV-2 nucleic acid by a throat swab.

The overall sample size in this meta-analysis was 7,467, of which 2,187 were in the severe COVID-19 cohort and 5,280 were in the non-severe COVID-19 cohort. The sample size for ALT was 1,957 (severe vs. non-severe $=566$ vs. 1,391), for AST: 2,107 (severe vs. non-severe $=612$ vs. 1495 ), for TBil: 1742 (severe vs. non-severe $=509$ vs. 1233), and for LDH: 1661 (severe vs. non-severe $=500$ vs. 1161 ), respectively.

The proportion of COVID-19 patients with abnormal ALT was $29 \%$. The proportion of severe and non-severe COVID-19 patients with abnormal ALT was $37.1 \%$ and $25.7 \%$, respectively. In terms of meta-analysis, significant heterogeneity existed (pooled $\mathrm{I}^{2}$ of OR was $70.4 \%$ ), thus we chose the randomeffects model. When compared between severe and non-severe COVID-19 patients, the pooled OR was 2.35 (95\% CI 1.383.98) (Fig 2-A).

The proportion of COVID-19 patients with abnormal AST was $29.2 \%$. The proportion of severe and non-severe COVID-19 patients with abnormal AST was $47.4 \%$ and $21.7 \%$, respectively. In terms of meta-analysis, the pooled $\mathrm{I}^{2}$ of OR was $21.2 \%$, thus we chose the fixed-effects model. When compared between severe and non-severe COVID-19 patients, the pooled OR was 3.21 (95\% CI 2.59-3.98) (Fig 2-B). 


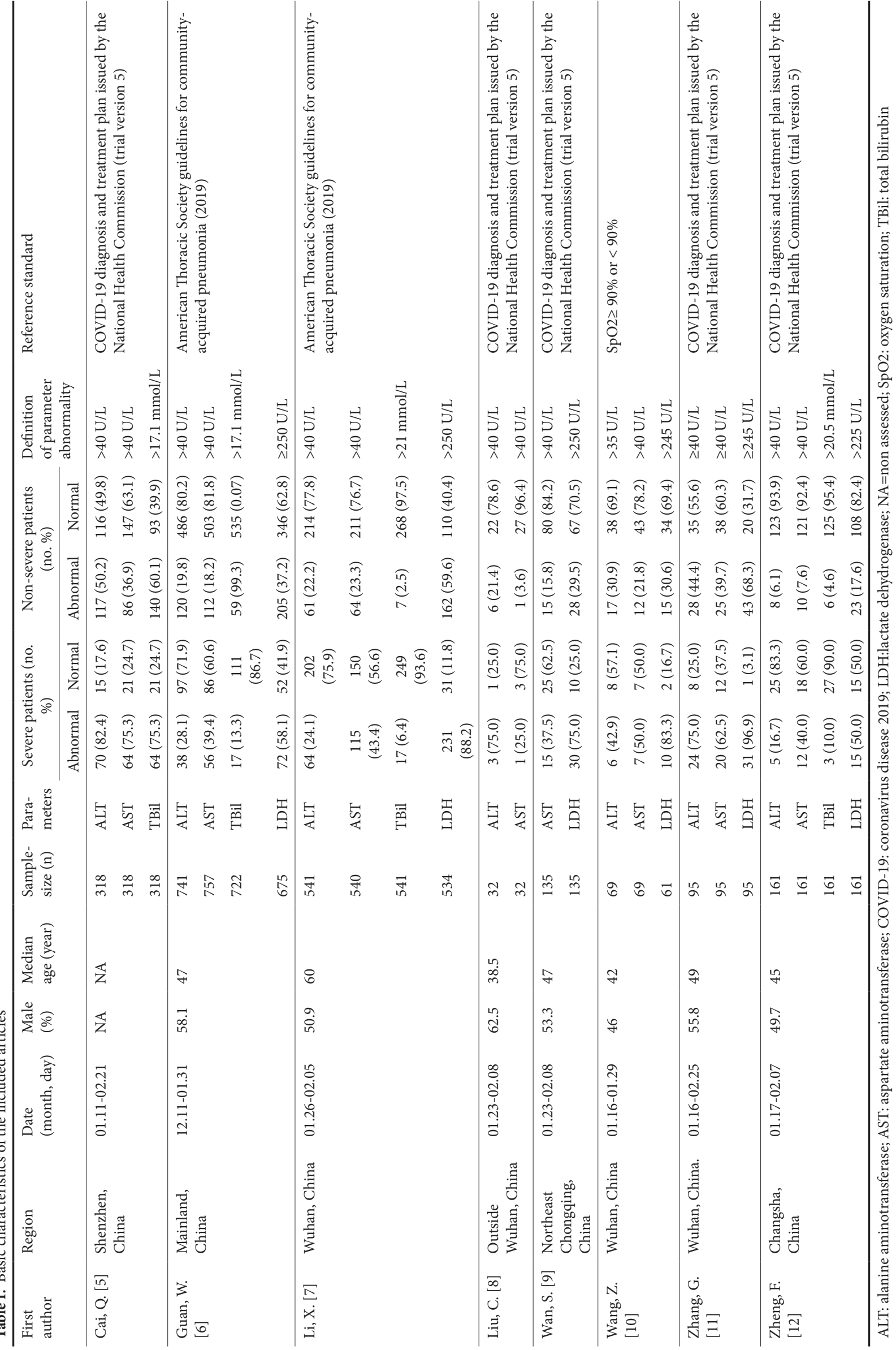




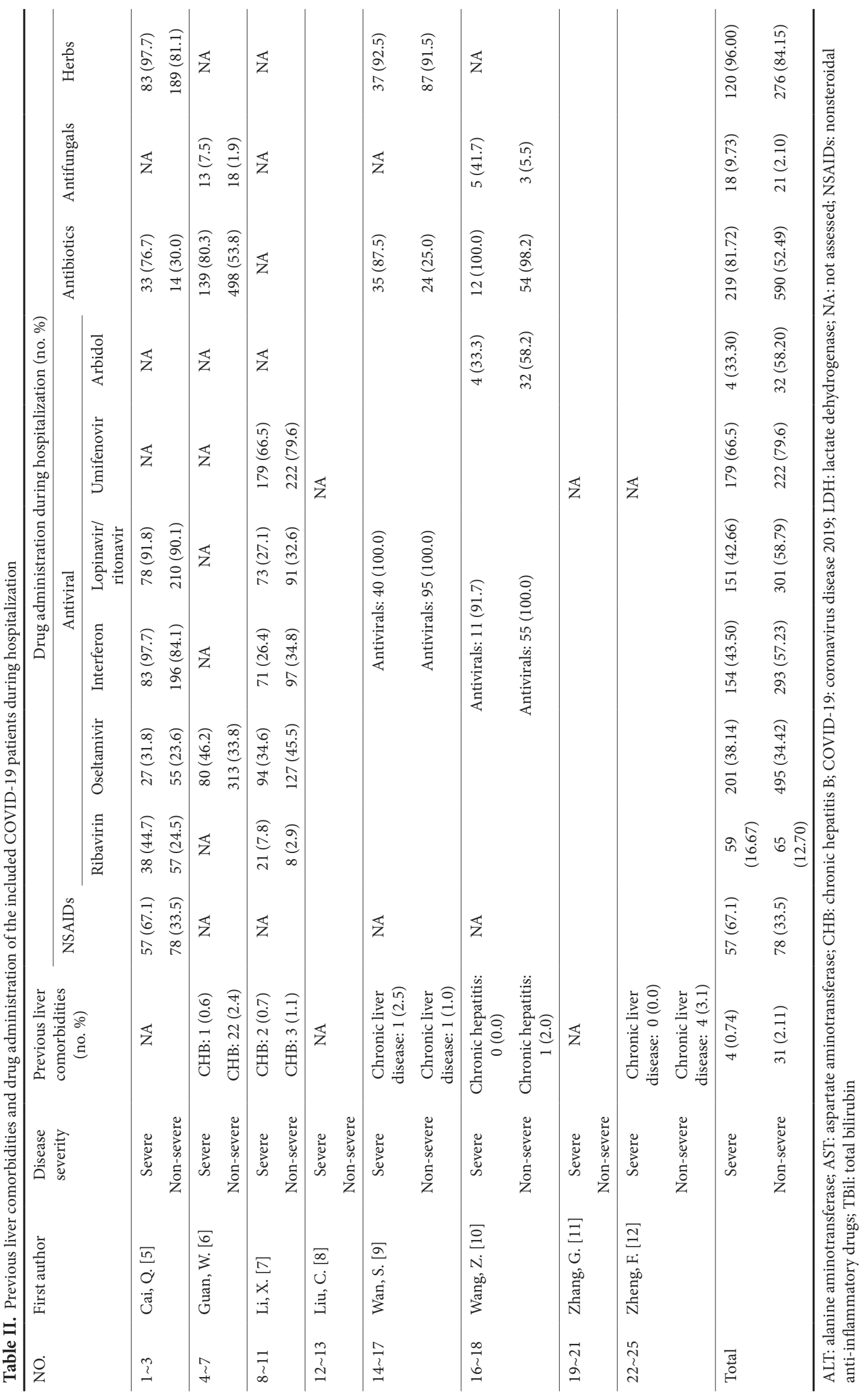


The proportion of COVID-19 patients with abnormal TBil was $18 \%$. The proportion of severe and non-severe COVID-19 patients with abnormal TBil was $19.8 \%$ and $17.2 \%$, respectively. In terms of meta-analysis, the pooled $\mathrm{I}^{2}$ of $\mathrm{OR}$ was $0 \%$, thus we chose the fixed-effects model. When compared between severe and non-severe COVID-19 patients, the pooled OR was 1.87 (95\% CI 1.32-2.65) (Fig. 2-C).

The proportion of COVID-19 patients with abnormal $\mathrm{LDH}$ was $52.1 \%$. The proportion of severe and non-severe COVID-19 patients with abnormal LDH was $77.8 \%$ and $41.0 \%$, respectively. For meta-analysis, significant heterogeneity existed (pooled $\mathrm{I}^{2}$ of OR was $62.1 \%$ ); thus we chose the random-effects model. When compared between severe and non-severe COVID-19 patients, the pooled OR was 4.83 (95\% CI 2.90-8.05) (Fig 2-D).

Collectively, liver damage was common in COVID-19 patients, especially in severe cases. The severity of COVID-19 can be a risk factor for abnormal liver function.

Based on the Harbord test, publication bias was not observed in the ALT test $(\mathrm{p}=0.135)$, AST test $(\mathrm{p}=0.192)$, TBil test $(p=0.492)$, and LDH test $(p=0.131)($ Fig 3$)$.

\section{DISCUSSION}

COVID-19 imposes a great challenge to public health around the world. The World Health Organization declared the novel coronavirus a "public health emergency of international concern". SARS-CoV-2 not only attacks the respiratory system but also impairs the myocardium, kidney, gastrointestinal tract and liver [13-17]. In this study, we systematically reviewed COVID-19-relevant liver injury; in particular we compared the risk of abnormal liver function tests between severe and non-severe COVID-19 patients. Eight articles were included in this meta-analysis with a total of 7,467 COVID-19 patients, comprising 2,187 severe cases and 5,280 non-severe cases. For severe patients, the risk of elevated ALT, AST, TBil, and LDH was 2.35 folds, 3.21 folds, 1.87 folds, and 4.83 folds higher than that in non-severe cases, respectively. In other words, severe COVID-19 patients were more susceptible to liver damage as compared with the non-severe ones. However, the reasons are manifold and complicated.

SARS-CoV-2 binding to the angiotensin-converting enzyme 2 (ACE2) receptor is the first step in the pathogenesis. Abundant ACE2 are located in alveolar type 2 epithelial cells. Therefore, the lung becomes the major target organ [18]. However, immunostaining and single cell sequencing showed that the ACE2 expression in liver was relatively low, and mainly located in the bile duct epithelial cells rather than hepatocytes [19-20]. Thus, we might speculate that ACE2 plays a minor role in COVID-19-relevant liver impairment. Also, damage to bile ducts lead to subsequent abnormality of hepatocytes. Cai et al. $[5,21]$ reported that $11.0 \%$ (severe vs. non-severe $=12.2 \%$ vs. $10.5 \%$ ) and $48.7 \%$ (severe vs. nonsevere $=75.3 \%$ vs. $39.1 \%$ ) of COVID-19 patients in Shenzhen, China presented elevated alkaline phosphatase (ALP) and $\gamma$-glutamyl transpeptidase (GGT) during hospitalization, respectively. However, compared with their baselines only $0.34 \%$ (severe vs. non-severe $=1.75 \%$ vs. $0 \%$ ) of the patients had higher ALP levels and 17.1\% (severe vs. non-severe = $43.1 \%$ vs. $10.8 \%$ ) of the cases had higher GGT levels during hospitalization. Therefore, we might speculate the damage to the bile duct occurs in the early stages of COVID-19, and bile
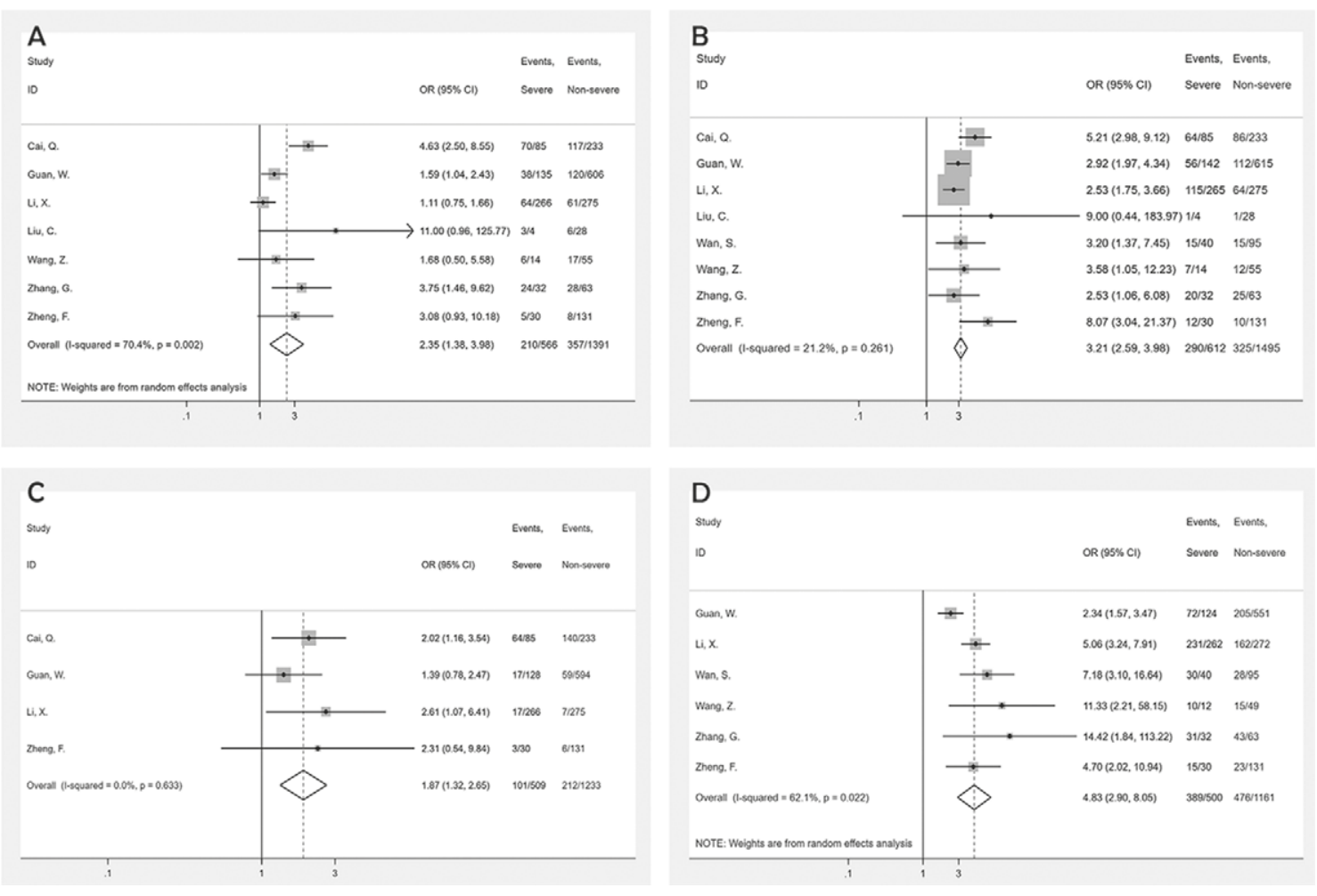

Fig. 2. The risk of abnormal ALT (A), AST (B), TBil (C), and LDH (D) level in severe COVID-19 patients compared to non-severe COVID-19 patients. ALT: alanine aminotransferase; AST: aspartate aminotransferase; COVID-19: coronavirus disease 2019; TBil: total bilirubin; LDH: lactate dehydrogenase 

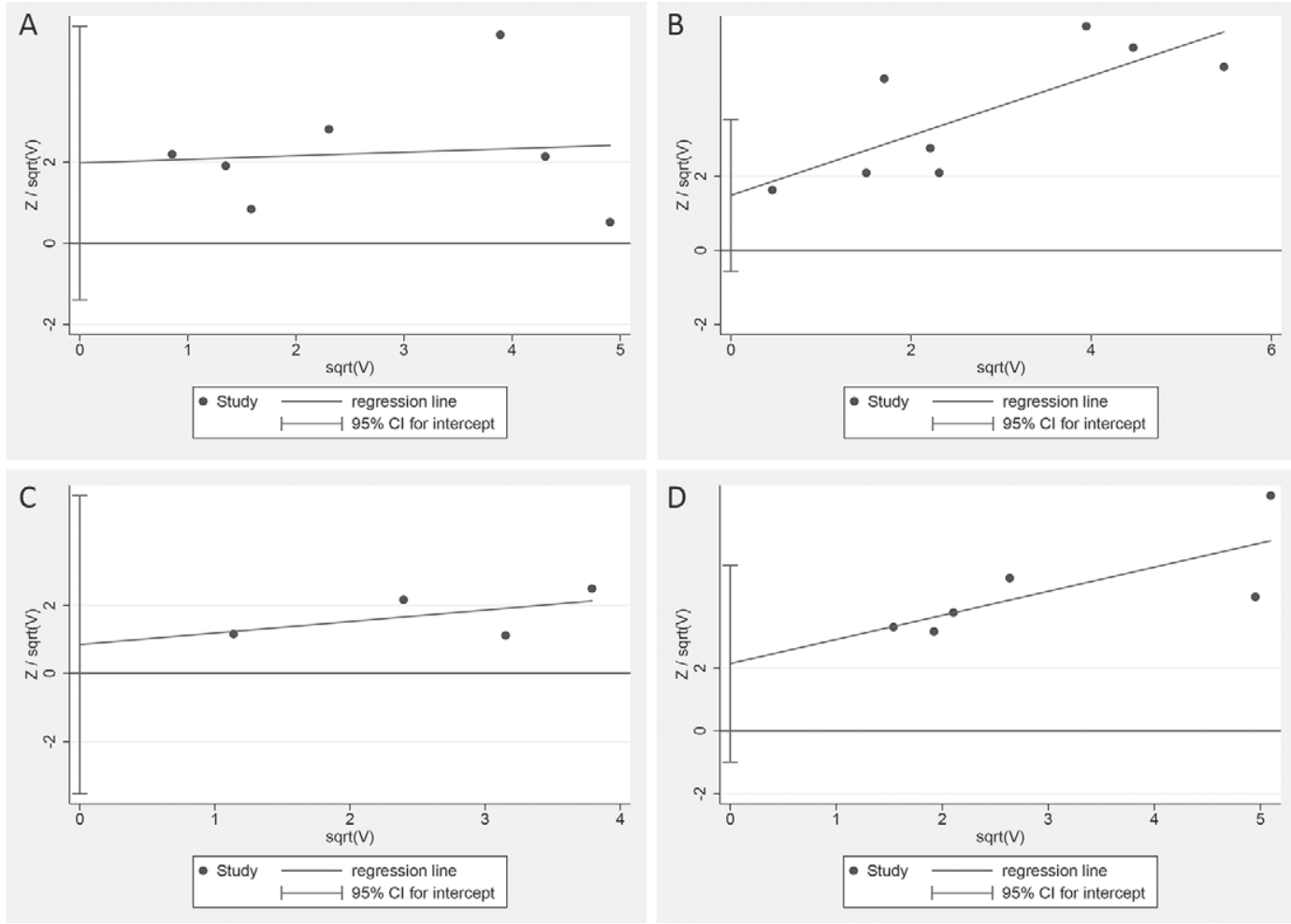

Fig. 3. Estimation of the publication bias for ALT (A), AST (B), TBil (C), LDH (D) test. ALT: alanine aminotransferase; AST: aspartate aminotransferase; COVID-19: coronavirus disease 2019; TBil: total bilirubin; LDH: lactate dehydrogenase

duct impairment was more common in severe COVID-19 patients. Inconsistently, other studies did not find abnormal ALT and GGT levels in COVID-19 patients [20, 22]. Thus, more research in this field is required.

Cytokine storms are involved in COVID-19 relevant liver injury. The cytokine storm is a systemic inflammatory response, caused by infection and trauma. A sharp increase in the level of a large number of pro-inflammatory cytokine can lead to an inflammatory cascade and uncontrolled tissue impairment [23]. The invasion of SARS-CoV-2 to host alveoli can trigger the innate and adaptive immune system, and a large amount of the pro-inflammatory factors are released, such as interleukin-6 (IL-6), which plays a key role in COVID-19 progression. A recent study published by Herold et al. [24] showed that IL- 6 can be used to predict respiratory failure in COVID-19 patients. A vast array of pro-inflammatory factors reaches the liver via circulation, which can activate Kupffer cells and leads to more IL- 6 release. IL- 6 triggers the vicious circle of nuclear factor (NF)- $\kappa$ B and STAT-3 signaling pathway and thereby induces a persistent inflammatory reaction in the liver [25]. In addition, signal transducer and activator of transcription (STAT)-3 can up-regulate expression of acute phase proteins in liver, which may in turn impair hepatocytes. Li et al. [7] investigated 312 COVID-19 patients in Wuhan, China, and found that $83.2 \%$ of the severe cases and $61.1 \%$ of the non-severe cases had elevated IL-6 levels $>7 \mathrm{ng} / \mathrm{L}$. Zhang et al. [11] reported that all the severe COVID-19 patients (oxygen saturation $<90 \%$ ) presented with the IL-6 levels $>20$ ng/L. Thereby, IL-6 is closely related to liver injury in severe COVID-19 patients.
Hypoxemia and hypoxic hepatitis represent another pathogenic mechanism. The current diagnosis of hypoxic hepatitis generally relies on three elements: a clinical setting resulting in reduced oxygen delivery or utilization by the liver, a significant increase in the serum aminotransferase level, and exclusion of other potential causes of liver injury [26]. Moreover, some studies found that respiratory failure patients with hypoxic hepatitis had low average levels of arterial oxygen but elevated central venous pressures, suggesting an element of hepatic venous congestion, which can cause further damage to the liver [27-29]. Apart from that, in septic shock, excessive endotoxin and inflammatory mediators can lead to an inability of hepatocytes to utilize oxygen [30-31]. A meta-analysis indicated that the incidence of hypoxic hepatitis was $2.5 \%$ in intensive care unit patients [32]. For severe COVID-19 patients, respiratory distress, hypoxemia and secondary bacterial infection are salient features. Therefore, severe COVID-19 patients are more likely to combine with hypoxic hepatitis. However, relevant studies are lacking at present.

Moderate microvascular steatosis with mild hepatic inflammation in a COVID-19 patient indicated the possibility of drug induced liver injury [33]. In our study, the patients used nonsteroidal anti-inflammatory drugs (NSAIDs), antivirals, antibiotics, antifungals, and herbs during hospitalization. All of these drugs could be potential agents to induce liver injury. Our study indicated that NSAIDs were more commonly taken among severe COVID-19 patients than non-severe patients (severe vs. non-severe $=67.1 \%$ vs. $33.5 \%$ ). In terms of antivirals, one study demonstrated that the risk of liver injury was in COVID-19 patients taking lopinavir/ritonavir than in 
patients who were not taking these drugs. [5]. Unexpectedly, in our study, lopinavir/ritonavir was more commonly used in non-severe COVID-19 patients rather than the severe patients (severe vs. non-severe $=42.66 \%$ vs. $58.79 \%$ ). Additionally, in our study, interferon, umifenovir and arbidol were more used in patients with non-severe COVID-19 and antibiotics, ribavirin, oseltamivir, herbs, and antifungals were more used in patients with severe COVID-19. In view of these inconsistent results, it is difficult to determine the effect of drugs as a confounder on liver damage during COVID-19 hospitalization. In view of these inconsistent results, more further studies on this aspect are needed. Compared with non-severe patients, severe patients would take drugs with wider varieties, higher dosage and longer treatment periods, which might enhance the risk of druginduced liver injury. When non-severe COVID-19 patients present with abnormal liver function tests, pharmacogenetic factors should be considered first $[20,22]$.

In our study, severe COVID-19 patients had a 3.21-fold and 4.83-fold higher risk of elevated AST and LDH than non-severe COVID-19 patients, respectively. Given that AST and LDH also exists in extrahepatic organs (e.g., myocardium), we speculate that extrahepatic diseases could also be important causes for abnormal AST and LDH in severe COVID-19 patients. Studies have observed significant distribution differences in underlying cardiovascular disease between severe and non-severe COVID-19 patients (severe vs. non-severe $=22.41 \%$ vs. $5 \%$ ), in particular in cardiac dysfunction grade $\geq 3$ (severe vs. non-severe $=10.4 \%$ vs. $0.4 \%$ ) [21]. Moreover, Li et al. [7] reported that the incidence of new-onset acute myocardial injury during hospitalization was higher among severe COVID-19 patients as compared with non-severe COVID-19 patients (severe vs. non-severe = $34.9 \%$ vs. 9.0\%). Apart from that, previous liver comorbidities play a complementary role in the abnormality of liver function parameters. The study of Cai et al. [21] demonstrated that severe COVID-19 patients were more likely to have chronic liver disease (including chronic hepatitis B, non-alcoholic fatty liver disease, and alcoholic liver disease) (severe vs. non-severe $=13.79 \%$ vs. $8.33 \%)$. For the patients with underlying liver disease, SARS$\mathrm{CoV}-2$ infection might be regarded as a "second hit" to the liver, which can trigger "acute-on-chronic" inflammation [20]. On the contrary, our study revealed that previous liver comorbidities were more common in non-severe COVID-19 patients rather than severe COVID-19 patients (severe vs. non-severe = $0.74 \%$ vs. $2.11 \%$ ). Therefore, further studies are required to determine whether previous liver comorbidities were significant confounding factors for COVID-19 relevant liver damage.

There are some limitations in this study. Because of the small number of studies, we were unable to perform subgroups analyses. The search strategy did not include all the MeSH terms for the liver enzymes, their abbreviations and synonyms. Bile duct injury associated parameters were not included in this study. Our meta-analysis was a descriptive research; we did not analysis the impact of abnormal liver parameters on the prognosis of COVID-19 patients. In view of the contextual realities in clinical practice, studies included in this meta-analysis cannot control the confounders for liver impairment such as drug administration and previous liver disease. Therefore, more studied are needed to affirm whether the disease severity is an independent risk factor for COVID-19-relevant liver damage.

\section{CONCLUSIONS}

Abnormal liver function tests are common in COVID-19 patients in mainland China, disease severity being associated with liver damage. Virus invasion, cytokine storm, hypoxic hepatitis, drug administration and co-morbidities might be involved in liver impairment during the course of COVID-19.

\section{Conflicts of interest: None to declare.}

Authors' contribution: S.L.X.: statistical analysis, data interpretation, and drafting the article. J.H.X. data interpretation. J.H.X., Y.Y.Y.: study design, data interpretation and critical revision of the manuscript.

Supplementary material: To access the supplementary material visit the online version of the J Gastrointestin Liver Dis at http://dx.doi. org/10.15403/jgld-2513

\section{REFERENCES}

1. Yang J, Zheng Y, Gou X, et al. Prevalence of comorbidities and its effects in patients infected with SARS-CoV-2: a systematic review and metaanalysis. Int J Infect Dis 2020;94:91-95. doi:10.1016/j.ijid.2020.03.017

2. Chen N, Zhou M, Dong X, et al. Epidemiological and clinical characteristics of 99 cases of 2019 novel coronavirus pneumonia in Wuhan, China: a descriptive study. Lancet 2020;395:507-513. doi:10.1016/S0140-6736(20)30211-7

3. Qian ZP, Mei X, Zhang YY, et al. Analysis of baseline liver biochemical parameters in 324 cases with novel coronavirus pneumonia in Shanghai area. Zhonghua Gan Zang Bing Za Zhi 2020;28:229-233. doi:10.3760/ cma.j.cn501113-20200229-00076

4. Zhu J, Ji P, Pang J, et al. Clinical characteristics of 3,062 COVID-19 patients: a meta-analysis. J Med Virol 2020 Apr 15. doi:10.1002/ jmv.25884

5. Cai Q, Huang D, Yu H, et al. COVID-19: Abnormal liver function tests. J Hepatol 2020 Apr 13. doi:10.1016/j.jhep.2020.04.006

6. Guan WJ, Ni ZY, Hu Y, et al. Clinical Characteristics of Coronavirus Disease 2019 in China. N Engl J Med 2020;382:1708-1720. doi:10.1056/ NEJMoa2002032

7. Li X, Xu S, Yu M, et al. Risk factors for severity and mortality in adult COVID-19 inpatients in Wuhan.J Allergy Clin Immunol 2020 Apr 20. doi:10.1016/j.jaci.2020.04.006

8. Liu C, Jiang ZC, Shao CX, et al. Preliminary study of the relationship between novel coronavirus pneumonia and liver function damage: a multicenter study. Zhonghua Gan Zang Bing Za Zhi 2020;28:107-111. doi:10.3760/cma.j.issn.1007-3418.2020.02.003

9. Wan S, Xiang Y, Fang W, et al. Clinical features and treatment of COVID-19 patients in northeast Chongqing. J Med Virol 2020 Mar 21. doi:10.1002/jmv.25783

10. Wang Z, Yang B, Li Q, Wen L, Zhang R. Clinical features of 69 cases with coronavirus disease 2019 in Wuhan, China.Clin Infect Dis 2020 Mar 16. doi:10.1093/cid/ciaa272

11. Zhang G, Zhang J, Wang B, Zhu X, Wang Q, Qiu S. Analysis of clinical characteristics and laboratory findings of 95 cases of 2019 novel coronavirus pneumonia in Wuhan, China: a retrospective analysis. Respir Res 2020;21:74. doi:10.1186/s12931-020-01338-8

12. Zheng F, Tang W, Li H, Huang YX, Xie YL, Zhou ZG. Clinical characteristics of 161 cases of corona virus disease 2019 (COVID-19) 
in Changsha. Eur Rev Med Pharmacol Sci 2020;24:3404-3410. doi:10.26355/eurrev_202003_20711

13. Han C, Duan C, Zhang S, et al. Digestive symptoms in COVID-19 patients with mild disease severity: clinical presentation, stool viral RNA testing, and outcomes. Am J Gastroenterol 2020 Apr 15. doi:10.14309/ ajg.0000000000000664

14. He XW, Lai JS, Cheng J, et al. Impact of complicated myocardial injury on the clinical outcome of severe or critically ill COVID-19 patients. Zhonghua Xin Xue Guan Bing Za Zhi 2020;48:E011. doi:10.3760/ cma.j.cn112148-20200228-00137

15. Pan L, Mu M, Yang P, et al. Clinical characteristics of COVID-19 patients with digestive symptoms in Hubei, China: a descriptive, cross-sectional, multicenter study. Am J Gastroenterol 2020;115:766-773. doi:10.14309/ ajg.0000000000000620

16. Shi S, Qin M, Shen B, et al. Association of cardiac injury with mortality in hospitalized patients with COVID-19 in Wuhan, China. JAMA Cardiol 2020 Mar 25. doi:10.1001/jamacardio.2020.0950

17. Xie H, Zhao J, Lian N, Lin S, Xie Q, Zhuo H. Clinical characteristics of non-ICU hospitalized patients with coronavirus disease 2019 and liver injury: A retrospective study. Liver Int 2020 Apr 2. doi:10.1111/ liv.14449

18. Cheng H, Wang Y, Wang GQ. Organ-protective effect of angiotensinconverting enzyme 2 and its effect on the prognosis of COVID-19. J Med Virol 2020 Mar 27. doi:10.1002/jmv.25785

19. Hamming I, Timens W, Bulthuis MLC, Lely AT, Navis GJ, van Goor H. Tissue distribution of ACE2 protein, the functional receptor for SARS coronavirus. A first step in understanding SARS pathogenesis. J Pathol 2004;203:631-637. doi:10.1002/path.1570

20. Li J, Fan JG. Characteristics and Mechanism of Liver Injury in 2019 Coronavirus Disease. J Clin Transl Hepatol 2020;8:13-17. doi:10.14218/ JCTH.2020.00019

21. Cai Q, Huang D, Ou P, et al. COVID-19 in a designated infectious diseases hospital outside Hubei Province, China. Allergy 2020 Apr 2. doi:10.1111/all.14309

22. Hu LL, Wang WJ, Zhu QJ, Yang L. Novel coronavirus pneumonia related liver injury: etiological analysis and treatment strategy.
Zhonghua Gan Zang Bing Za Zhi 2020;28:97-99. doi:10.3760/cma.j.is sn.1007-3418.2020.02.001

23. Zhang $\mathrm{C}, \mathrm{Wu} \mathrm{Z}, \mathrm{Li}$ JW, Zhao H, Wang GQ. The cytokine release syndrome (CRS) of severe COVID-19 and Interleukin-6 receptor (IL-6R) antagonist Tocilizumab may be the key to reduce the mortality. Int J Antimicrob Agents 2020;55:105954. doi:10.1016/j. ijantimicag.2020.105954

24. Herold T, Jurinovic V, Arnreich C, et al. Level of IL-6 predicts respiratory failure in hospitalized symptomatic COVID-19 patients. medRxiv 2020 Apr 10. doi:10.1101/2020.04.01.20047381

25. $\mathrm{He} \mathrm{G}$, Karin M. NF- $\mathrm{KB}$ and STAT3 - key players in liver inflammation and cancer. Cell Res 2011;21:159-168. doi:10.1038/cr.2010.183

26. Waseem N, Chen PH. Hypoxic Hepatitis: A review and clinical update. J Clin Transl Hepatol 2016;4:263-268. doi:10.14218/ JCTH.2016.00022

27. Birrer R, Takuda Y, Takara T. Hypoxic hepatopathy: pathophysiology and prognosis. Intern Med 2007;46:1063-1070. doi:10.2169/ internalmedicine.46.0059

28. Henrion J, Schapira M, Luwaert R, Colin L, Delannoy A, Heller FR. Hypoxic hepatitis: clinical and hemodynamic study in 142 consecutive cases. Medicine (Baltimore) 2003;82:392-406. doi:10.1097/01. md.0000101573.54295.bd

29. Sun J, Aghemo A, Forner A, Valenti L. COVID-19 and liver disease. Liver Int 2020 Apr 6. doi:10.1111/liv.14470

30. Nelson DP, Samsel RW, Wood LD, Schumacker PT. Pathological supply dependence of systemic and intestinal $\mathrm{O} 2$ uptake during endotoxemia J Appl Physiol 1988;64:2410-2419. doi:10.1152/jappl.1988.64.6.2410

31. Zhang H, Vincent JL. Oxygen extraction is altered by endotoxin during tamponade-induced stagnant hypoxia in the dog. Circ Shock 1993;40:168-176.

32. Tapper EB, Sengupta N, Bonder A. The incidence and outcomes of ischemic hepatitis: a systematic review with meta-analysis. Am J Med 2015;128:1314-1321. doi:10.1016/j.amjmed.2015.07.033

33. Xu Z, Shi L, Wang Y, et al. Pathological findings of COVID-19 associated with acute respiratory distress syndrome. Lancet Respir Med 2020;8:420-422. doi:10.1016/S2213-2600(20)30076-X 